\title{
mmTrace: Modeling Millimeter-wave Indoor Propagation with Image-based Ray-tracing
}

\author{
Daniel Steinmetzer \\ TU Darmstadt, Germany \\ dsteinmetzer@seemoo.tu-darmstadt.de
}

\author{
Jiska Classen \\ TU Darmstadt, Germany \\ jclassen@ seemoo.tu-darmstadt.de
}

\author{
Matthias Hollick \\ TU Darmstadt, Germany \\ mhollick@seemoo.tu-darmstadt.de
}

\begin{abstract}
Current mm-wave indoor propagation analysis techniques have limited options when it comes to more than one transmitter and receiver. Experimental testbed hardware is expensive and state-of-the-art simulation methods, such as statistical channel models, are limited to specific scenarios. To overcome these problems, we present mmTrace, a fast deterministic image-based ray-tracing simulation framework for $\mathbf{m m}$-wave propagation. It supports developing mm-wave specific protocols and, in contrast to common statistical models, deals with multiple transceivers. The strengths of mmTrace constitute signal variations at different receivers and interference of multiple transmitters, which are crucial in certain situations. We implement our framework in MATLAB and validate simulated channel impulse responses against those of statistical channel models for IEEE 802.11ad in well-defined scenarios. Our results indicate that image-based ray-tracing is a feasible tool to predict interference in $\mathbf{m m}$-wave communication systems.
\end{abstract}

\section{INTRODUCTION}

Millimeter-wave (mm-wave) communication systems address the demands of $5 \mathrm{G}$ networks: efficiency, low latency, and very high throughput [1]. On strongly directional transmission links, signals are steered directly to their destination with low overshoot. Numerous new application scenarios emerge; therefore, comprehensive models are required to understand the specialties of mm-wave propagation. The statistical channel models of [2] provide a foundation to analyze $\mathrm{mm}$ waves propagating in common environments. Such models predict channels between two nodes precisely, but have their shortcomings. They neither adapt to complex environments nor consider multiple transceivers, which are crucial for a good protocol design. We need better models to predict mm-wave propagation in situations such as dense networks with highly directional links.

Ray-tracing techniques can handle multiple transceivers and therewith determine receiver correlations and transmitter interference. Yet, indoor channels strongly depend on the precise layout of the environment [3] - in particular for mm-waves, which reflect on most surfaces and behave quasi-optically [4]. Even though efficient ray-tracing techniques exist [5], to the best of our knowledge, no practical mm-wave ray-tracing tool that focuses on different antenna settings and multiple transceivers is currently available.

We propose mmTrace, a mm-wave ray-tracing framework implemented in MATLAB to predict the channel impulse response (CIR) in specific environments between multiple transceivers. Interference analysis, as illustrated in Figure 1a,

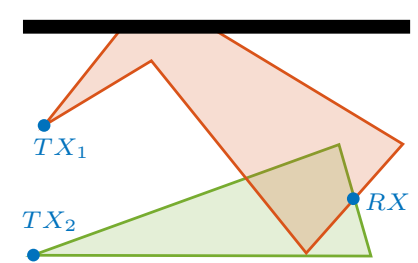

(a) Interfering transmitters

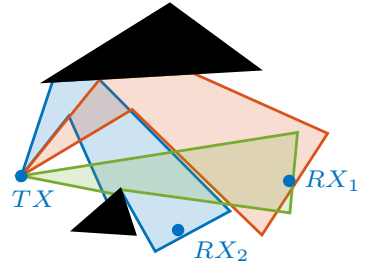

(b) Multiple receivers
Fig. 1. Scenarios with multiple transmitters or receivers

becomes a simple addition of two signals. Similarities and differences between two receivers that overhear the same signal over independent paths as sketched in Figure $1 \mathrm{~b}$ are also measurable. The effects in such situations highly depend on the antenna orientation and cannot be obtained from statistical models. We use image-based ray-tracing to determine multipath channels between transceivers over multiple reflections, since this approach is more efficient than the common shooting and bouncing rays (SBR) technique. We consider position, orientation, and antenna characteristics of transceivers placed in a $2 \mathrm{D}$ environment with reflecting and blocking obstacles. Although we neglect diffraction, scattering, and vertical propagation for computational performance, our results adequately approximate the precision of statistical techniques. The special strength of mmTrace is its capability to adapt to different scenarios and physical environments and deal with multiple transceivers in parallel. Our implementation is publicly available [6] and provides a fundamental simulation tool to analyze various aspects of mm-wave propagation.

The remainder of this paper is structured as follows. In Section II we discuss related work and methods. Our mm-wave ray-tracing framework mmTrace is described in Section III. We validate mmTrace against the statistical model of [2] in Section IV and demonstrate its performance in Section V. Finally, we conclude our work in Section VI.

\section{RELATED WORK}

Simulations of mm-wave propagation can be performed with statistical or deterministic models. While the former use measurement statistics from concrete scenarios, the latter draws on physical theories confirmed by measurements.

\section{A. Channel Measurements}

Early measurements of mm-waves in the $60 \mathrm{GHz}$ domain are taken in [7]. On short distances, weather effects and 
atmospheric absorptions have little effect [8] but human blockage can be severe [9]. Diffraction is less relevant for $\mathrm{mm}$ waves than in lower frequencies [10]. Interference in $\mathrm{mm}$ wave networks might be a more limiting factor than noise [11]. Consumer grade antenna arrays exhibit strong side-lobes that potentially cause interference [12]. However, the authors of [13] confirm that mm-wave transmissions are feasible over reflected signal paths. All these works outline the fundamental different aspects of mm-waves that need to be considered in propagation models.

\section{B. Statistical Propagation Models}

Statistical models allow for fast computation. They typically utilize statistics obtained from physical measurements in specific environments. A seminal statistical model was investigated in [14] and assumes multipath components to arrive in clusters. Amplitudes of the signal components within each cluster follow a Rayleigh or Rician distribution. In either case, the phases of received signals are uniformly distributed. The channel models for IEEE 802.11 [15] adapt statistical predictions to WiFi based multiple-input and multiple-output (MIMO) communication systems in the $2.4 \mathrm{GHz}$ band. Their MATLAB implementation provides a fundamental design and analysis tool for WiFi low layer aspects [16]. The indoor simulation tool SIRCIM predicts statistical channel impulse responses [17] in line-of-sight (LOS) and non-line-of-sight (NLOS) scenarios. It is designed for mm-waves at $60 \mathrm{GHz}$ but also works for lower frequencies. Their model produces realistic multipath channel characteristics with high precision. A complete $60 \mathrm{GHz}$ statistical channel model for typical indoor environments is established in [10], confirming the quasioptical nature of $\mathrm{mm}$-waves and negligibility of diffraction. The model in [2] specifically predicts the channel statistics for IEEE 802.11 ad networks. This is similar to earlier 802.11 models [15] but also considers directional antennas, variable beamwidth, and antenna orientation. Unfortunately, statistical propagation models are limited to scenarios similar to those in the underlaying measurements. Detailed empirical analyses are required for comprehensive models.

\section{Deterministic Propagation Models}

Deterministic models base on the theory of electromagnetic wave propagation that could be solved with Maxwell's equations. Unfortunately, this approach is impractical because of high computational requirements. However, as mm-wave propagation behaves quasi-optically [18], ray-tracing becomes a practical approximation.

Ray-tracing can be divided into shooting and bouncing rays (SBR), and image-based methods. SBR methods intuitively launch rays in many directions, which are reflected by objects and potentially hit the receiver. For a large number of rays, SBR can reach high precision and encounter diffraction and scattering. In contrast to SBR, image-based ray-tracing only considers direct reflections. Receiver locations are mirrored on the objects surfaces.

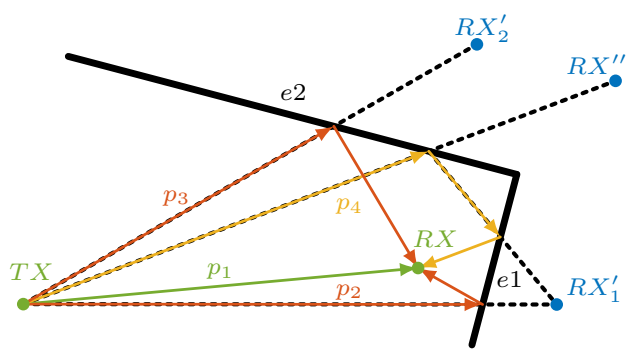

Fig. 2. Paths based on image-based ray-tracing.

The feasibility of mm-wave ray-tracing to identify 2D clusters of arrivals is verified in [3], which indicates that raytracing provides precise results for scatter-free LOS scenarios. Measurements in [4] confirm that image-based ray-tracing can determine the majority of multipath components in mm-wave transmissions. Moreover, calculating first-order and secondorder reflections is sufficient for LOS applications [4]. A hybrid approach leveraging both, SBR- and image-based methods, is proposed in [5]. It is compared to real-world measurements in a meeting room but reveals small deficits in time domain predictions. Nevertheless, image-based ray-tracing provides an efficient tool for coarse grained channel modeling.

\section{MMTRACE}

Our framework for mm-wave signal propagation modeling in indoor environments, mmTrace, is separated in three stages to allow for independent processing and comparison of intermediate results with other models:

1) trace paths between transceivers,

2) extract channels from paths, and

3) characterize the extracted channels.

Each of these stages is described in the following, before we validate and evaluate our approach in the subsequent sections.

\section{A. Trace Paths Between Transceivers}

To determine paths between transceivers surrounded by obstacles in a 2D environment, we apply the image-based raytracing approach. Consider two transceivers TX and RX and two reflecting walls represented by the edges $e_{1}$ and $e_{2}$ as illustrated in Figure 2. After determining the first order images $\mathrm{RX}_{1}^{\prime}$ and $\mathrm{RX}_{2}^{\prime}$ of $\mathrm{RX}$ in $e_{1}$ and $e_{2}$, we determine the second order image of $\mathrm{RX}_{1}^{\prime}$ in $e_{2}$ resulting in $\mathrm{RX}^{\prime \prime}$. We construct the paths using edge intersections towards the images but ignore paths that miss the reflecting edge. Doing so, we obtain the first order reflection paths $p_{2}$ and $p_{3}$, and the second order reflection path $p_{4}$ in addition to the LOS path $p_{1}$. In mmTrace this is implemented recursively. Assuming that the room and all obstacles are represented by polygons, we express each of them as a set of reflecting edges. To decrease computation time we filter for valid reflectors that are not completely blocked in each recursion step. Thereby, we construct the paths between two transceivers as a geometrical problem.

\section{B. Extract Channels from Paths}

The channel representation is obtained by assigning each path a complex amplitude gain and delay based on environmental properties. We compute the delay as $\tau=d / c_{0}$, where 
$d$ is the length of the path including reflections and $c_{0}$ the speed of light. The complex amplitude gain $h_{k}$ of path $k$ is compound from multiple properties: the transmitter and receiver antenna gain, the reflection coefficient, and the path attenuation.

1) Antenna Gains: radiation patterns describe antenna gains towards particular directions. In mmTrace, we implement different radiation patterns shown in Figure 3a: (1) an omnidirectional pattern, (2) a directional pattern, and (3) a side-lobe pattern. The simplest model is the omni-directional pattern with a constant gain in all directions, which is typically applied for lower frequency systems. The directional pattern pushes the radiation towards a certain direction in which a very high gain is achievable. We utilize the directional antenna pattern as specified in [19] in dependency of the half-power beamwidth (HPBW). We further model the maximum gain according to [2], which leads to varying gains of different directionality as illustrated in Figure 3b. The side-lobe pattern exhibits an exemplary, non-ideal antenna gain that consists of a main-lobe in the indented direction, a back-lobe in the opposite direction, and several side-lobes in arbitrary directions. We define this as the sum of multiple directional radiation patterns with angle offset and variable strength. However, to be independent from specific antennas, mmTrace supports the integration of custom radiation patterns.

2) Reflection Coefficients: assuming each object has a constant complex permittivity, we compute reflections using Fresnel's equations as a function of this permittivity and the angle-of-incidence. The reflection coefficient is the product of the phase shifts and attenuations that affect the signal through all reflections on a path and depends on the objects' permittivity. As [5] already found out, validating realistic model assumptions to characterize reflections on particular objects is challenging. Providing realistic models that exactly match physical environments is beyond the scope of this paper. mmTrace is a framework supporting different reflection characteristics with arbitrary permittivity.

3) Path Attenuation: besides the effects of antenna radiation and reflection loss, signals are also affected by attenuation and phase shift while propagating through air. The attenuation is expressed by the free-space path loss (FSPL), which defines the gain of a received signal dependent on the propagating distance $d$, and wavelength $\lambda$. The phase shift is considered as a rotation by $d / \lambda$ in the complex plane.

\section{Characterize the Channel}

From the channel representation in the previous stage, we extract metrics that describe different characteristics of the channel shown in Table I. In particular, we derive the channel impulse response (CIR), which is the most common model to describe channel effects in time domain. It is the sum of Dirac impulses, representing the delay, and power of all multipath components. The power delay profile (PDP) is the squared CIR magnitude and describes the distribution of the channel power over time. The mean delay describes the average time of arrival of the earliest significant multipath component, while the root

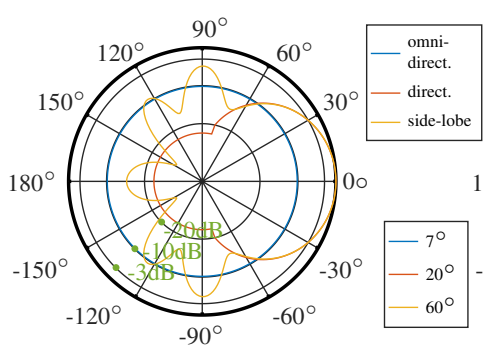

(a) Radiation patterns

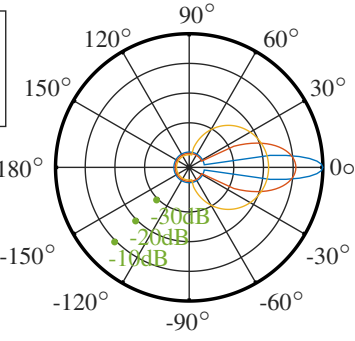

(b) Impact of beamwidths
Fig. 3. Radiation patterns implemented in mmTrace

mean square (RMS) delay spread expresses its variation. The cumulative channel power is the sum of the channel power of all multipath components.

\section{VALIDATION}

We validate our mm-wave propagation model by comparing simulated CIRs of mmTrace with those obtained from the statistical model in [2]. We use the MATLAB implementation of the statistical model in [19], and adjust the properties for our 2D environment by neglecting reflections on the floor and ceiling. We rebuild the empty $4.5 \times 3.0 \mathrm{~m}$ conference room and $7.0 \times 7.0 \mathrm{~m}$ living room environments from [2] in mmTrace. In the conference room, transceivers are randomly located on a $2.5 \times 1.0 \mathrm{~m}$ table in the center with a distance of $2 \mathrm{~m}$. The living room represents a typical scenario for wireless entertainment systems, as the receiver is located close to the middle of one wall. The transmitter takes an arbitrary location, $4 \mathrm{~m}$ away from the receiver. To characterize wall reflections we use the permittivity of $2.26-0.24 j \mathrm{E}-3$ as experimentally determined in [20]. All antennas use the directional radiation pattern with $60^{\circ}$ or $20^{\circ} \mathrm{HPBW}$. At all positions, we examine 64 antenna orientations. In the conference room, we distinguish between LOS and NLOS scenarios. We repeat all simulations 1000 times to state the median and $95 \%$ confidence bounds.

In the following, we characterize the channels obtained from both models, demonstrate the feasibility of ray-tracing for channel modeling, and analyze the impacts of NLOS and higher communication distances.

\section{A. Channel Characterization}

To characterize the channels in both models, we consider the conference room scenario with antennas of both transceivers

TABLE I

APPLIED METRICS FOR CHANNEL CHARACTERIZATION

\begin{tabular}{ll}
\hline Channel Characteristic: & Mathematical Expression: \\
\hline \hline Channel impulse response (CIR): & $h(\tau)=\sum_{k=1}^{K} h_{k} \delta\left(\tau-\tau_{k}\right)$ \\
Power delay profile (PDP): & $p(\tau)=|h(\tau)|^{2}$ \\
mean delay: & $\bar{\tau}=\frac{\sum_{k=1}^{K}\left|h_{k}\right|^{2} \tau_{k}}{\sum_{k=1}^{K}\left|h_{k}\right|^{2}}$ \\
RMS delay spread: & $\tau_{\mathrm{RMS}}=\sqrt{\frac{\sum_{k=1}^{K}\left(\tau_{k}-\bar{\tau}\right)^{2}\left|h_{k}\right|^{2}}{\sum_{k=1}^{K}\left|h_{k}\right|^{2}}}$ \\
cumulative channel power: & $P=\sum_{k=1}^{K}\left|h_{k}\right|^{2}$ \\
\hline
\end{tabular}



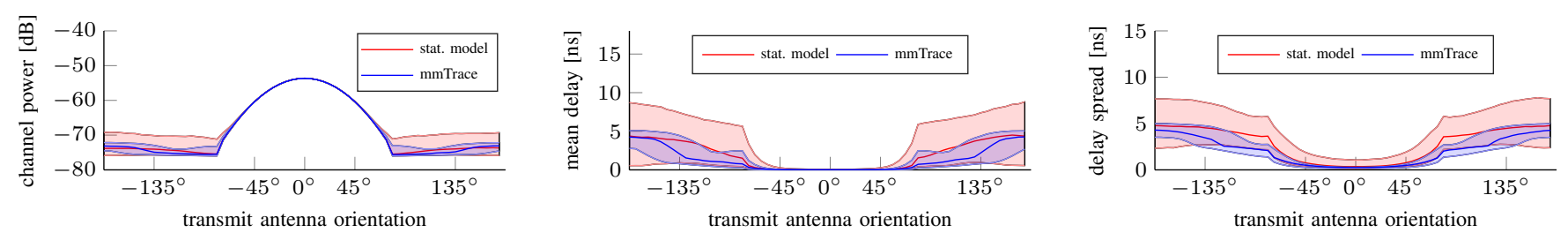

(a) Conference room scenario with LOS paths and $60^{\circ}$ beamwidth
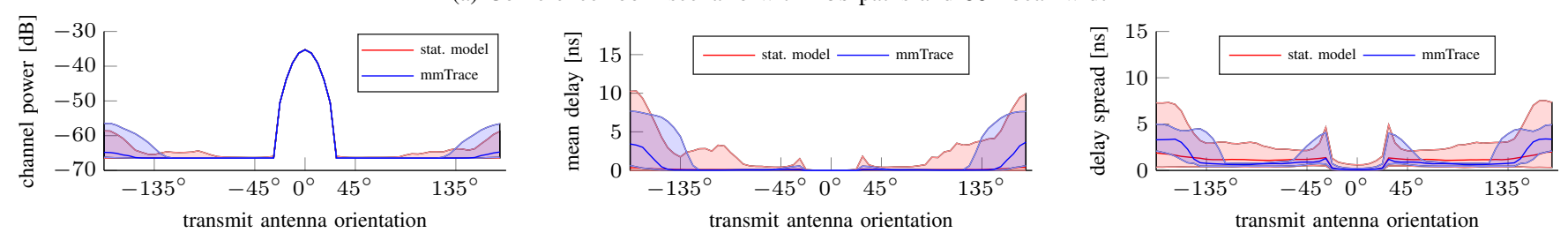

(b) Conference room scenario with LOS paths and $20^{\circ}$ beamwidth
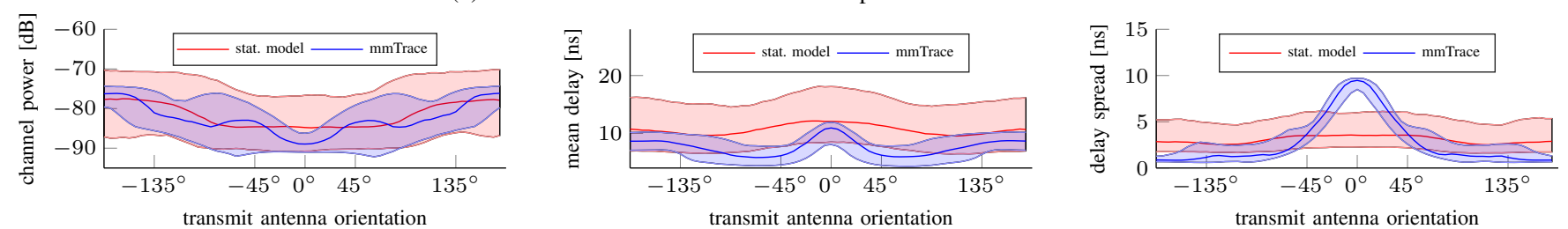

(c) Conference room scenario without direct path and $60^{\circ}$ beamwidth
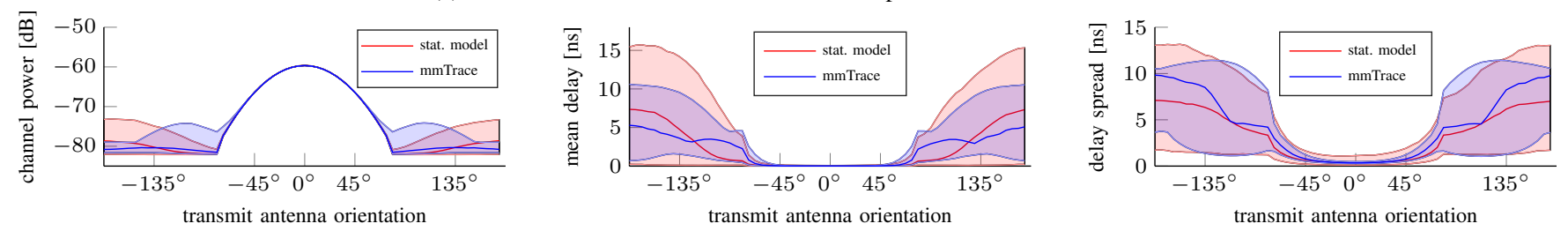

(d) Living room scenario with LOS paths and $60^{\circ}$ beamwidth

Fig. 4. Channel characteristics in different scenarios with rotating transmitter. The median and the $95 \%$ confidence bounds of 1000 simulations are depicted.

directly oriented towards each other. We simulate the CIRs and extract the PDPs as shown in Figure 5 for 10 simulations. At first sight, both results significantly differ. Only the high power peak at zero delay perfectly matches. For higher delays, the CIR of mmTrace exhibits only single impulses that correspond to certain reflection paths. The statistical model has smoother impulses which come from clustering effects and scattering that is not encountered in mmTrace. However, especially the paths with $30 \mathrm{~ns}$ delay show that mmTrace predicts the intensity of channel paths similarly to the statistical channel models.

Figure 6 shows the distribution of received power over the reflection order in mmTrace. In LOS scenarios (Figure 6a), more than $99 \%$ of power comes over the direct path. In NLOS scenarios (Figure 6b), multi-order reflections experience increased relevance. First order reflections provide $79 \%$ of power, $17 \%$ come over second order reflections. Reflection paths with higher order than four barely contribute to the received power. While the statistical model considers up to two reflections, we limit the maximum number of reflections to four in mmTrace in all further experiments.

\section{B. Feasibility of mmTrace}

Analyzing the conference room scenario with LOS and halfpower beamwidth (HPBW) of $60^{\circ}$ as shown in Figure $4 \mathrm{a}$

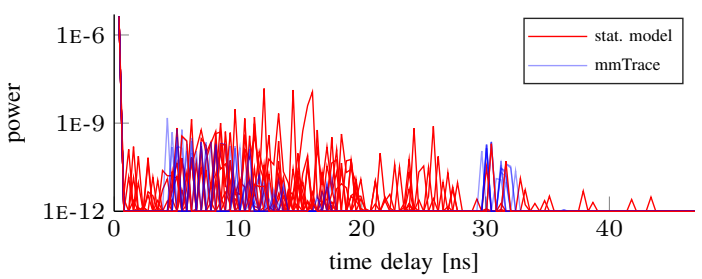

Fig. 5. Power delay profiles of mmTrace and the statistical model in the conference room scenario at 10 random positions.

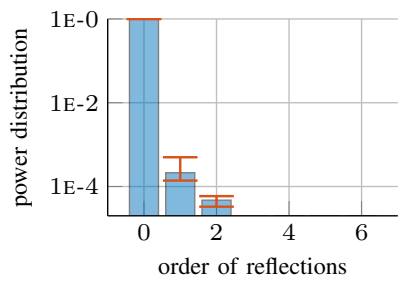

(a) A LOS scenario

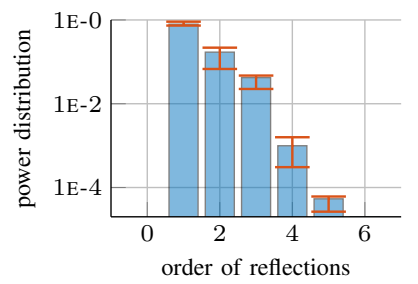

(b) A NLOS scenario
Fig. 6. Distribution of received signal strength (RSS) over reflection order.

confirms the feasibility of mmTrace. The cumulative channel power perfectly matches within the HPBW. Only for misaligned antennas, the statistical model has a slightly higher confidence bound, because we use distinct paths rather than statistical probabilities. This observation extends through all 


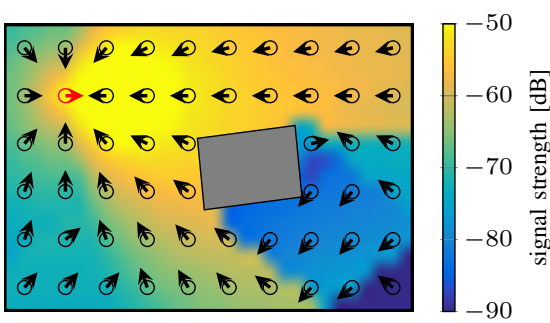

(a) Received signal from first transmitter.

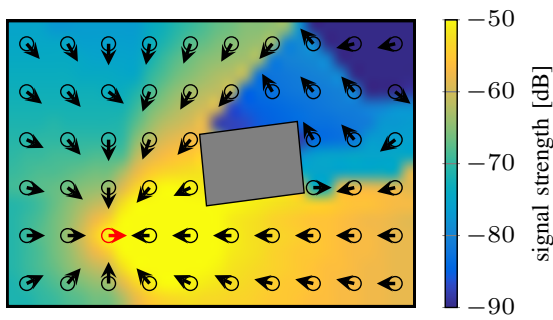

(b) Received signal from second transmitter.

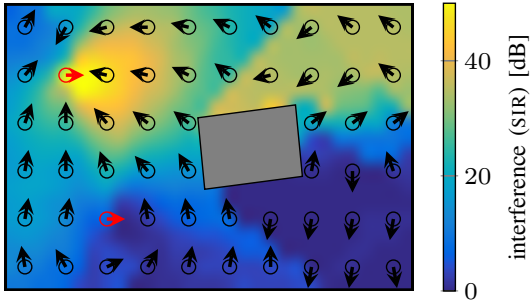

(c) Interference from second transmitter.

Fig. 7. RSS and interference with ideal radiation pattern and $60^{\circ} \mathrm{HPBW}$. Arrows indicate optimal alignment. Transmitters are red, receivers black.

experiments. We also observe a lower bound for the RSS that is caused by a constant side-lobe level in the applied radiation pattern. The mean delay and RMS delay spread also match within the HPBW. For a small misalignment, mmTrace slightly underestimates the delay but approaches the statistical model again when the transmitter orientates to the opposite direction. Nevertheless, the differences are marginal.

Narrowing the HPBW to $20^{\circ}$, as shown in Figure 4b, mainly decreases the mean delay and RMS delay spread in both models. Only for a strong misalignment, a high delay remains recognizable. In this situation mmTrace slightly overestimates compared to the statistical model, but the median still falls into the model's confidence bounds.

\section{Blockage in the direct path}

In NLOS scenarios, where the direct path is blocked, we obtain the results shown in Figure $4 \mathrm{c}$ for $60^{\circ} \mathrm{HPBW}$. The channel power is significantly lower than in LOS scenarios and remains almost independent from the antenna orientation. While the statistical model provides smooth transitions of all three parameters, our results exhibit little power peaks corresponding to the concrete reflection paths. For antenna misalignment, mmTrace predicts a lower mean delay and RMS delay spread. For aligned antennas, both parameters significantly grow, which is not observable for the statistical model. However, only the delay spread in mmTrace exceeds the confidence bounds of the statistical model.

\section{Impact of Increased Distances}

With increased distances, as in the living room scenario, we identify significantly higher delays than in the conference room scenario as shown in Figure $4 \mathrm{~d}$ for $60^{\circ} \mathrm{HPBW}$ and LOS. This effect obviously comes from the longer reflection paths. Due to the receivers position and the larger room, similar powered reflections arrive from multiple directions that contribute to a higher delay. These results show that mmTrace successfully adapts to this situation: channel power, mean delay, and RMS delay spread are very close to the statistical channel model.

\section{Evaluation}

So far, we only validated mmTrace against the statistical approach and were able to show comparable results with few discrepancies. In this section, we aim at demonstrating the advantages of our approach. In particular, we present application scenarios including multiple interfering transceivers that are impossible to investigate with statistical channel models.

We assume a room sized $5.0 \times 3.0 \mathrm{~m}$ with a blocking and reflecting wooden obstacle. Again, we use the permittivities taken from [20]: $2.26-0.24 j \mathrm{E}-3$ for walls and $2.8-4 j \mathrm{E}-2$ for the wooden obstacle. Two transmitters are oriented in parallel to cause interferences. Received signals are obtained throughout the room. Unless noted otherwise, all antennas use the directional radiation pattern with $60^{\circ} \mathrm{HPBW}$. In total, we obtain CIRs at 540 locations for 64 antenna orientations. We assume that all receivers can flexibly adjust their antenna orientation and steer it for optimal results. As mentioned in Section IV-A, we limit the maximum number of reflections to four. In the following, we analyze reception properties at multiple receiver positions, consider interference of multiple transmitters, and finally switch to the more realistic side-lobe antenna patterns.

\section{A. Mapping the received signal strength}

Figure $7 \mathrm{a}$ shows the map of reception capabilities with optimal antenna alignment for the first transmitter (red). The background color indicates the achievable RSS while the arrows indicate the direction for optimal orientation. At all positions roughly inside the signal beam, receiving antennas directly steer towards the transmitter. At locations behind the blocking objects or far out of the beam, receivers orient towards first order reflections. We identify very sharp transitions between certain areas. At the transitions, even small distances between the receivers lead to completely different orientations. Yet a small location offset might have a significant impact on the channel.

\section{B. Interfering Transmitters}

For the second transmitter, we observe a similar pattern, as shown in Figure 7b. Only the shadow region moves to the other side of the obstacle. We consider the signal of this transmitter as interference and calculate the signal-to-interference ratio (SIR) as $\mathrm{SIR}=P_{\mathrm{TX} 1} / P_{\mathrm{T} \times 2}$, where $P_{\mathrm{TX} 1}$ and $P_{\mathrm{TX} 2}$ are the received signal strengths from both transmitters respectively. The SIRs in optimal antenna orientation are shown in Figure 7c. Receivers aim at steering their antenna towards the first transmitter but away from the second transmitter. Therefore, even some receivers in LOS of the first transmitter misalign their antennas to suppress interference. The optimal orientation 


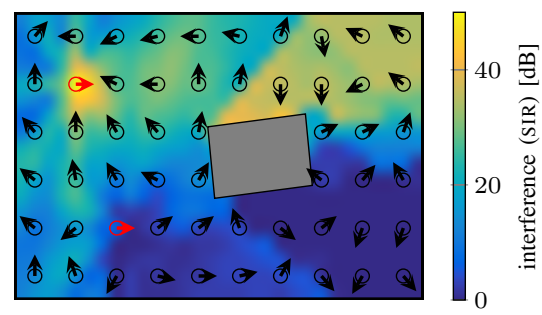

Fig. 8. Interference with custom side-lobe radiation pattern and $60^{\circ} \mathrm{HPBW}$. Arrows indicate optimal alignment. Transmitters are red, receivers black.

might change from the non-interfering scenario. But receivers are affected differently: some regions still exhibit a suitable signal quality while others completely interfere.

\section{Interference in Side Lobes}

So far, we only considered the ideal directional radiation pattern with a single lobe. The side-lobe pattern defined in Section III-B1 leads to different RSS values. In the interference scenario in Figure 8 receivers not only try to steer their main lobe towards the first transmitter, they also aim at keeping away their side-lobes from the interfering signal. This can have interesting effects even on the receivers in the shadow region behind the object that blocks the interfering signal: receivers tend to orientate their antenna completely away from the transmitter to overcome interference in the side-lobes. In the shadow region, antenna orientations appear random. This shows that in case of interference, antenna orientations for best SIR do not necessarily correspond to the LOS direction even though a direct path exists.

\section{CONCLUSION}

Statistical channel models are the state-of-the-art to predict propagation effect. However, they reach their limits when dealing with highly directional communication. Steerable antennas and distinct reflections on environmental objects lead to significant channel variations at low location offset. Nonideal antenna radiation patterns lead to complex interference scenarios. Environmental properties that were negligible for omni-directional communication systems take an important role for mm-waves.

In this work, we present a mm-wave indoor propagation modeling framework based on deterministic image-based raytracing to simulate channel properties for specific antennas and environments with multiple transceivers. An implementation is publicly available at [6]. By neglecting diffraction and scattering we cannot achieve the precision of statistical channel models, but provide a suitable approximation. Our validation results reveal only low discrepancies. Simulating precise propagation characteristics is challenging due to inaccurate environmental models. We agree with [5] that basic propagation characteristics can be well modeled by ray-tracing with little inaccuracies. Nevertheless, our framework provides valuable benefit through its capability to adjust to different physical scenarios and consider multiple transmissions in parallel.
Dealing with interference in dense networks is essential to develop reliable and efficient protocols for mm-wave systems.

\section{ACKNOWLEDGMENT}

This work has been funded by the DFG within CROSSING, the BMBF and the State of Hesse within CRISP-DA, and the Hessian LOEWE excellence initiative within CASED.

\section{REFERENCES}

[1] L. Young, "Telecom experts plot a path to 5G," IEEE Spectrum, vol. 52, no. $10,2015$.

[2] A. Maltsev, "Channel models for $60 \mathrm{GHz}$ WLAN systems," Tech. Rep. doc.: IEEE 802.11-09/0334r8, 2010.

[3] B. Neekzad, K. Sayrafian-Pour, J. Perez, and J. Baras, "Comparison of ray tracing simulations and millimeter wave channel sounding measurements," in IEEE 18th International Symposium on Personal, Indoor and Mobile Radio Communications, 2007.

[4] H. Xu, V. Kukshya, and T. Rappaport, "Spatial and temporal characteristics of $60-\mathrm{GHz}$ indoor channels," IEEE Journal on Selected Areas in Communications, vol. 20, no. 3, 2002.

[5] W. Peter, W. Keusgen, and R. Felbecker, "Measurement and ray-tracing simulation of the $60 \mathrm{GHz}$ indoor broadband channel: model accuracy and parameterization," in The Second European Conference on Antennas and Propagation., 2007.

[6] D. Steinmetzer. (2016) mmTrace: ray-tracing based millimeterwave propagation simulation. [Online]. Available: https://github.com/ seemoo-lab/mmTrace

[7] P. Smulders and A. Wagemans, "Wideband indoor radio propagation measurements at $58 \mathrm{GHz}$, Electronics Letters, vol. 28, no. 13, 1992.

[8] T. Manabe, Y. Miura, and T. Ihara, "Effects of antenna directivity on indoor multipath propagation characteristics at $60 \mathrm{GHz}$," Sixth IEEE International Symposium on Personal, Indoor and Mobile Radio Communications, 1995.

[9] S. Singh, F. Ziliotto, U. Madhow, E. Belding, and M. Rodwell, "Blockage and directivity in $60 \mathrm{GHz}$ wireless personal area networks: from cross-layer model to multihop MAC design,' IEEE Journal on Selected Areas in Communications, 2009.

[10] A. Maltsev, R. Maslennikov, A. Sevastyanov, A. Lomayev, A. Khoryaev, A. Davydov, and V. Ssorin, "Characteristics of indoor millimeter-wave channel at $60 \mathrm{GHz}$ in application to perspective WLAN system," in Proceedings of the Fourth European Conference on Antennas and Propagation, 2010.

[11] H. Shokri-Ghadikolaei and C. Fischione, "Millimeter wave ad hoc networks: noise-limited or interference-limited?" IEEE Global Communications Workshop, 2015.

[12] T. Nitsche, G. Bielsa, I. Tejado, A. Loch, and J. Widmer, "Boon and bane of $60 \mathrm{GHz}$ networks: practical insights into beamforming, interference, and frame level operation," International Conference on emerging Networking EXperiments and Technologies, 2015.

[13] J. Ansari, N. Perpinias, A. Nahring, P. Mahonen, and M. Petrova, "Empirical characterization of mm-wave communication links in realistic indoor scenarios," in Wireless Communications and Networking Conference, 2015

[14] A. Saleh and R. Valenzuela, "A statistical model for indoor multipath propagation," IEEE Journal on Selected Areas in Communications.

[15] "TGn channel models," Tech. Rep. doc.: IEEE 802.11-03/940r4, 2004.

[16] "IEEE 802.11n channel models." [Online]. Available: mathworks.com/ help/comm/examples/ieee-802-11n-channel-models.html

[17] T. Rappaport, S. Seidel, and K. Takamizawa, "Statistical channel impulse response models for factory and open plan building radio communicate system design," IEEE Transactions on Communications, vol. 39, no. 5, 1991.

[18] A. Maltsev, R. Maslennikov, A. Sevastyanov, A. Khoryaev, and A. Lomayev, "Experimental investigations of $60 \mathrm{GHz}$ WLAN systems in office environment," IEEE Journal on Selected Areas in Communications, 2009.

[19] R. Maslennikov and A. Lomayev, "Implementation of $60 \mathrm{GHz}$ WLAN channel model ," Tech. Rep. doc.: IEEE 802.11-10/0854r3, 2010.

[20] J. Lu, D. Steinbach, P. Cabrol, P. Pietraski, and R. V. Pragada, "Propagation characterization of an office building in the $60 \mathrm{GHz}$ band," The 8th European Conference on Antennas and Propagation, 2014. 